\title{
Desenvolvimento de lodo granular aeróbio em reatores em batelada sequencial com baixa velocidade de sedimentação
}

\author{
Development of aerobic granular sludge in \\ sequencing batch reactors with low sedimentation speed
}

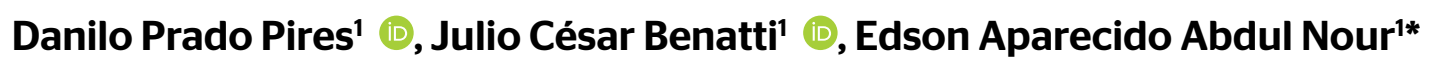

\begin{abstract}
RESUMO
Diversos trabalhos têm mostrado a formação de lodo granular aeróbio em reatores nos quais são impostas elevadas velocidades de sedimentação, da ordem de 10 a 12 m.h.'. Aparentemente, quando a velocidade de sedimentação é inferior a 3,8 m.h'1', a fração de lodo floculado é predominante, visto que o lodo suspenso não éeliminado de forma efetiva do reator. Outros estudos, entretanto, mostram a formação de lodos granulares aeróbios para velocidades menores que essa, apontando a possibilidade da formação desse tipo de biomassa em velocidades ainda menores. Assim, este trabalho avaliou a formação desse tipo de lodo em reatores que tratam esgoto sanitário, com relação altura/diâmetro unitária, para velocidades de sedimentação de 1,8 e 1,2 m.h-1 , verificando as eficiências de remoção de matéria orgânica e nitrogênio. Os resultados obtidos indicaram que é possível formar lodo aeróbio granular para a faixa de velocidade de estudo, porém com baixa estabilidade estrutural para diâmetros de 1,2 mm ou mais. Essa instabilidade dos grânulos contribui para a baixa eficiência de remoção de matéria orgânica e nitrogênio nos reatores.
\end{abstract}

Palavras-chave: lodo granular aeróbio; velocidade de sedimentação; relação h/d; esgoto doméstico.

\begin{abstract}
Several studies have shown the formation of aerobic granular sludge in reactors where high sedimentation rates are imposed, varying from 10 to $12 \mathrm{~m} . \mathrm{h}^{-1}$. Apparently, when the settling velocity is less than $3.8 \mathrm{~m} \cdot \mathrm{h}^{-1}$, the fraction of flocculated sludge is predominant, since the suspended sludge is not effectively eliminated from the reactor. Other studies, however, show the formation of aerobic granular sludge at velocities lower than this, pointing to the possibility of formation of this type of biomass at even lower speeds. Thus, this work evaluated the efficacy of this type of sludge in reactors treating sewage, with $\mathrm{H} / \mathrm{D}$ ratio (height/diameter), for sedimentation velocities of 1.8 and $1.2 \mathrm{mh}^{-1}$, verifying the efficiencies of organic matter and nitrogen. The results indicated that it is possible to form aerobic granular sludge for the study velocity range, but with low structural stability from diameters of approximately $1.2 \mathrm{~mm}$. This instability of the granules contributes to the low efficiency of removal of organic matter and nitrogen in the reactors.
\end{abstract}

Keywords: aerobic granular sludge; settling velocity; h/d ratio; domestic sewage.

\section{INTRODUÇÃO}

Adav e Lee (2008) definem os grânulos aeróbios como agregados com linhagens de microrganismos funcionais envoltos por uma matriz de substâncias poliméricas extracelulares (EPS), compostos inorgânicos e minerais. A utilização dessa tecnologia, nova e promissora, permite a concepção de plantas de tratamento de esgoto compactas em virtude da capacidade que esses grânulos têm para remover simultaneamente matéria orgânica e nutrientes em um único reator em batelada sequencial (RBS) (DE KREUK, 2007).

De acordo com Liu e Tay (2004), existem diversos fatores que influenciam na formação dos lodos granulares aeróbios, como a composição do substrato e sua taxa de aplicação, as forças de cisalhamento hidrodinâmicas, o regime feast-famine (limitação de substrato), a estratégia de enchimento do reator, a concentração de oxigênio dissolvido, a configuração do reator e sua relação altura/ diâmetro (H/D), a idade do lodo, o tempo de ciclo, o tempo de sedimentação e a relação de troca volumétrica (RTV). Entretanto, segundo Liu et al. (2005), a velocidade de sedimentação e a RTV são as principais variáveis que induzem à formação do lodo granular aeróbio (LGA).

Sengar et al. (2018) afirmam que a chave para a formação dos grânulos aeróbios é a pressão de seleção, induzida pelo baixo tempo de sedimentação. O tempo de sedimentação é função da velocidade de sedimentação e da distância

\section{$\square$}

'Universidade de Campinas - Campinas (SP), Brasil.

*Autor correspondente: ednour@fec.unicamp.br

Conflitos de interesse: os autores declaram não haver conflitos de interesse.

Financiamento: nenhum.

Recebido: 15/05/202O - Aceito: 19/11/202O - Reg. ABES: 20200180 
até o ponto de descarga do reator. Liu et al. (2005), analisando os dados de Qin et al. (2004) e Wang (2005), afirmam que a fração dos grânulos aeróbios no total do lodo de um reator aumenta linearmente com o incremento da velocidade de sedimentação, porém, quando a velocidade é inferior a $3,8 \mathrm{~m} \cdot \mathrm{h}^{-1}$, a fração de lodo floculado é predominante.

Por outro lado, Awang e Shaaban (2016), ao estudarem parâmetros cinéticos de lodos granulares aeróbios, mostram a formação desse tipo de lodo em reatores com velocidades de sedimentação baixas, de 5,4 e 3,0 m.h.' e relação H/D de 11,3 e 4,4, respectivamente. Apesar de o tempo de start up ser maior para o reator com menor velocidade de sedimentação, os autores sublinham o desempenho eficiente desse reator quando comparado ao de maior velocidade.

Corsino et al. (2016) indicam a necessidade do desenvolvimento de LGA em reatores de relação H/D menor, proporcionando baixas velocidades de sedimentação. Os autores relatam que, em escala real, o uso de reatores do tipo coluna é limitado em razão das elevadas alturas necessárias.

Dessa forma, parece ser possível a formação de lodos granulares aeróbios em reatores com velocidades de sedimentação bastante baixas, em reatores com baixa relação H/D, pois, como afirma Pujol (2008), a velocidade de sedimentação dos grânulos é maior do que a dos flocos. Assim, este trabalho visa verificar a formação desse tipo de lodo em reatores com velocidades de sedimentação

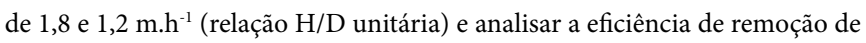
matéria orgânica e nitrogênio, utilizando esgoto doméstico.

\section{METODOLOGIA}

\section{Concepção dos reatores}

Os reatores utilizados neste trabalho foram instalados no Laboratório de Protótipos de Tratamento de Águas e Efluentes (LABPRO) da Faculdade de Engenharia Civil, Arquitetura e Urbanismo da Universidade Estadual de Campinas (FEC/ UNICAMP). Na Figura 1 é apresentado o layout dos reatores RBS 1 e RBS 2, ambos com volume útil de $8 \mathrm{~L}$, e RTV de 75 e 50\%, respectivamente.
A aeração dos reatores foi realizada por cortina de difusão de ar (marca Boyu de $105 \mathrm{~cm}$ ), tendo sua vazão de ar controlada por um rotâmetro (marca Dwyer, modelo de Fluxo de 1-10 SCFM de ar). Utilizou-se a vazão de ar de $4 \mathrm{~L}$. $\mathrm{min}^{-1}$, seguindo as indicações de Show et al. (2012). Considerando-se essa taxa de aeração e a área transversal do reator, obtém-se a velocidade ascensional do ar de $0,17 \mathrm{~cm} . \mathrm{s}^{-1}$. O valor de velocidade ascensional, apesar de estar abaixo dos valores tipicamente utilizados nesse tipo de sistema (STURM \& IRVINE, 2008), estão de acordo com estudos nos quais se aplicam baixas cargas orgânicas (HE et al., 2016; HE et al., 2017; HE et al., 2018; LI et al., 2019; ROCKTÄSCHEL et al., 2015). A taxa de aeração aplicada foi de $4 \mathrm{~L} \cdot \mathrm{min}^{-1}$, seguindo as indicações de Show et al. (2012) e Li et al. (2019), para quem a intensidade de aeração deve estar entre os valores de 2,0 a 6,0 L ar.min ${ }^{-1}$, pois elevadas taxas de aeração atrapalham a formação do lodo granular. O suprimento de ar comprimido foi mantido por compressor do tipo pistão. A operação dos reatores foi feita por automação utilizando-se temporizadores digitais (marca Brasfort ${ }_{0}$ ), válvulas solenoides (marcas Aicás, Werk e Smart Solution,), sensor de nível (marca Icos, LA12N-40) e relé de interface (marca Jng, Jar50).

\section{Operação dos reatores}

O esgoto utilizado nos ensaios, com composição de um esgoto sanitário característico, foi gerado na área norte do campus da UNICAMP, onde estão localizados laboratório de ensino e pesquisa, salas de aula, bancos, escolas, restaurantes, lanchonetes e o Hospital de Clínicas. A coleta era feita diariamente, diretamente da rede, e o esgoto era conduzido para um reservatório que alimentava os reatores.

Os reatores operaram em ciclos de 480 min (8 horas), divididos em seis etapas: enchimento, reação anaeróbia (com mistura mecânica), reação aeróbia sedimentação, descarte e repouso, como ilustrado na Figura 2.

$\mathrm{O}$ experimento foi dividido em três fases. A fase 1 foi realizada visando à aclimatação dos microrganismos e teve duração de 98 dias (14 semanas), com o tempo de sedimentação de $50 \mathrm{~min}$. Iniciou-se pela inoculação dos reatores com lodo proveniente de um experimento alimentado com o mesmo esgoto sanitário. A fase 2 foi realizada com o intuito de induzir a proliferação dos
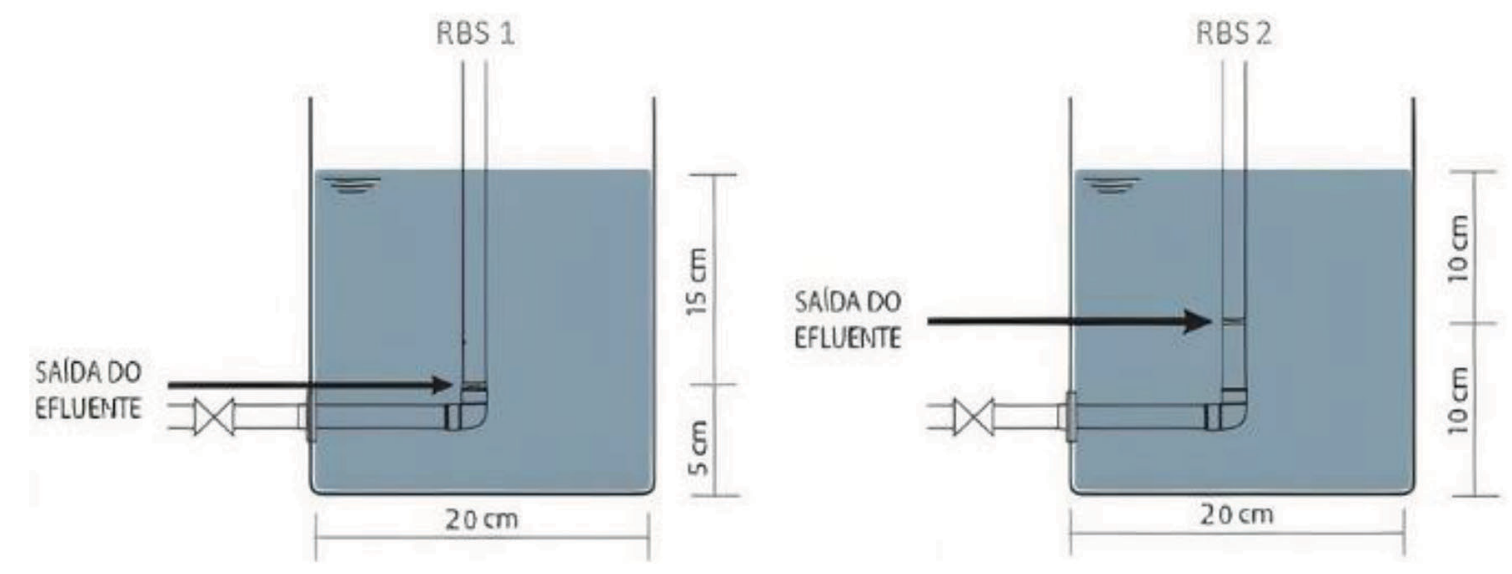

RBS: reator em batelada sequencial.

Fonte: elaborada pelos autores.

Figura 1 - Layout dos reatores em batelada sequencial 1 e 2. 
microrganismos formadores de grânulos no interior dos reatores e teve 126 dias (18 semanas). Nessa fase, o tempo de sedimentação foi reduzido em 5 min a cada 14 dias, tempo esse que foi acrescentado na etapa de reação aeróbia até se atingir o tempo de sedimentação final de 5 min. A diminuição do tempo de sedimentação foi baseada nos trabalhos de Su et al. (2013), Wagner et al. (2015) e Liu et al. (2017). Na fase 3 os reatores foram operados com tempo de sedimentação de 5 min ao longo de cinco semanas ( 35 dias).

Na Figura 3 são apresentadas as velocidades de sedimentação impostas ao longo de cada fase do experimento. A velocidade de sedimentação do LGA varia de $25 \mathrm{a} 70 \mathrm{~m} \cdot \mathrm{h}^{-1}$, sendo ele vantajoso para operar reatores mais compactos e com maior fluxo hidráulico (SHOW et al., 2012). Todavia, os pesquisadores Liu et al. (2010) afirmam que, em RBS alimentados com esgoto sanitário de baixa carga orgânica, o tempo de formação para o processo de granulação ser concluído é maior (tendo sido de 400 dias no trabalho citado). Dessa forma, o tempo de operação e a carga aplicada devem ser levados em consideração para os baixos valores da velocidade de sedimentação.

\section{MÉTODOS ANALÍTICOS}

Na Tabela 1 são apresentados os métodos e a frequência das análises realizadas para o monitoramento das características físicas e químicas do afluente, liquor misto e efluente dos reatores, bem como os ensaios utilizados para a caracterização da biomassa.

Para acompanhar as características da formação dos grânulos e verificar o diâmetro do lodo granular, foram feitas análises morfológicas do lodo semanalmente, em todas as fases. Utilizando a metodologia aplicada por Jungles (2011), as amostras dos lodos dos reatores foram colocadas em uma lâmina de

\begin{tabular}{|c|c|c|}
\hline 2 & 3 & $4 x_{0}^{0} 6$ \\
\hline \multicolumn{3}{|c|}{ 17 Etapa 1 : enchimento $(15 \mathrm{~min}) ; \quad 2$ Etapa 2 : anaeróbia $(60 \mathrm{~min}) ;$} \\
\hline \multicolumn{3}{|c|}{3 Etapa 3 : aeróbia ( 330 a 375 min); 4 Etapa 4 : sedimentação (50 a 5 min) } \\
\hline 5 Eta & : descarte (15 min); & 6 Etapa 6 : repouso $(10 \mathrm{~min})$ \\
\hline
\end{tabular}

Figura 2 - Etapas de operação dos reatores em batelada sequencial 1 e 2.

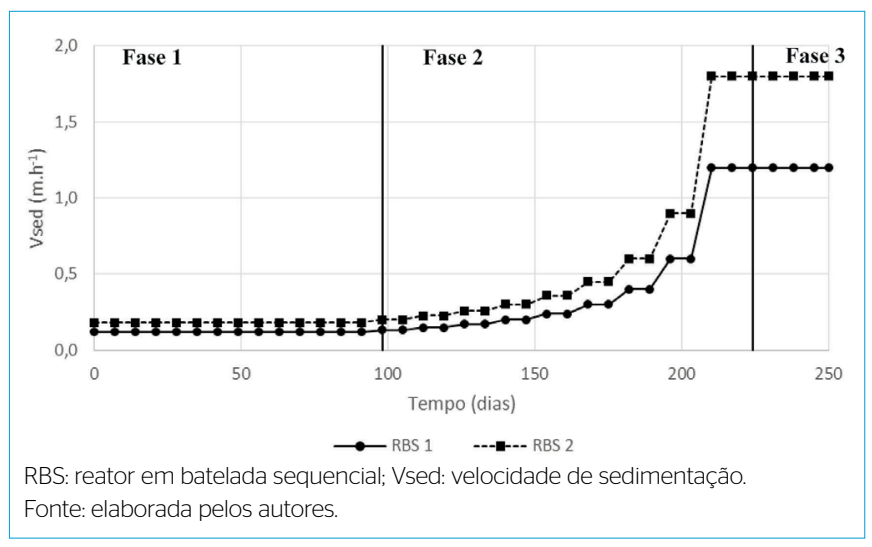

Figura 3 - Velocidade de sedimentação para cada uma das fases do experimento. vidro e dispostas em um microscópio com sistema digital de captura (NIKON, modelo E 200). Após a captura, os procedimentos de análise das imagens para a caracterização do lodo foram realizados com o software Image J, baseados no artigo de Bueno et al. (2013).

A EPS é excretada pelos próprios microrganismos e ajuda na adesão celular, dando a estabilidade necessária para a atividade dos grânulos dentro do reator. Para extrair essa substância, seguiram-se os métodos de extração aplicado nos artigos de Adav e Lee (2008) e Liu e Fang (2002), em que foi utilizada a extração por meio de formaldeído e hidróxido de sódio (Formaldeído$\mathrm{NaOH}$ ), considerada a mais eficiente na remoção da EPS quando comparada aos outros métodos químicos e físicos. Grande parte da composição da EPS é formada de uma fração de carboidratos (polysaccharides - PS) e proteínas (proteins - PN), sendo necessária a utilização de métodos como o do ácido fenol-sulfúrico (NIELSEN, 2010) e de Lowry (1945), respectivamente, para quantificar essas substâncias.

A velocidade de sedimentação do tipo zonal foi medida com base na metodologia de Von Sperling (2012), a qual consiste em relacionar a velocidade de sedimentação em zona com a concentração do lodo, observando com o passar do tempo uma diferença entre as fases líquida e sólida.

\section{APRESENTAÇÃO E ANÁLISE DOS RESULTADOS}

\section{Características do afluente}

$\mathrm{Na}$ Tabela 2, a seguir, são apresentados os dados da caracterização físico-química do afluente.

Verificam-se, com base na análise da Tabela 2, elevados valores de desvio padrão para as variáveis analisadas. Esse fato deve-se às peculiaridades do esgoto gerado na universidade, com grande influência dos horários letivos e fins de semana. Além disso, há períodos em que o principal contribuinte na rede é o Hospital de Clínicas, o que aumenta ainda mais a heterogeneidade das amostras.

\begin{tabular}{|c|c|c|}
\hline Análise & Método & Frequência \\
\hline Oxigênio dissolvido & Standard methods* 4500 OG & Diária \\
\hline Temperatura & Standard methods* 2550 B & Diária \\
\hline $\mathrm{pH}$ & Standard methods* $4500 \mathrm{HB}$ & Diária \\
\hline Alcalinidade & Standard methods* $2320 \mathrm{~B}$ & Semanal \\
\hline DQO & Standard methods* $5220 \mathrm{D}$ & Semanal \\
\hline Nitrogênio amoniacal & Standard methods* $4500 \mathrm{NH} 3 \mathrm{C}$ & Semanal \\
\hline Nitrogênio total Kjeldahl & Standard methods* 4500 & Semanal \\
\hline Série de sólidos & $\begin{array}{c}\text { Standard methods* } 2540 \mathrm{E} \\
\text { 2540D 2540F }\end{array}$ & Semanal \\
\hline IVL & Schwarzenbeck et al. (2004) & Semanal \\
\hline Diâmetro médio da biomassa & Jungles (2011) & Semanal \\
\hline EPS & Adav e Lee (2008) & Semanal \\
\hline $\begin{array}{l}\text { Velocidade de sedimentação } \\
\text { zonal }\end{array}$ & Von Sperling (2012) & Semanal \\
\hline
\end{tabular}

DQO: demanda química de oxigênio; IVL: índice volumétrico de lodo; EPS: substâncias poliméricas extracelulares.

Fonte: APHA (2005). 
O afluente analisado pode ser classificado como de média a baixa carga orgânica e de elevada concentração de nitrogênio amoniacal (METCALF \& EDDY, 2013). Entretanto, isso se torna um ponto vantajoso, pois trabalhos originais devem ser elaborados para descobrir inovações na forma de granular, utilizando efluentes reais de baixa carga orgânica (NANCHARAIAH \& KIRAN KUMAR REDDY, 2018).

\section{Comportamento da biomassa}

A concentração de sólidos suspensos voláteis (SSV) no liquor misto e efluente de saída dos reatores é apresentada na Figura 4. Verifica-se uma tendência no aumento da concentração da biomassa no interior dos reatores na fase inicial (fase 1), seguida de instabilidade ao longo da fase 2, quando se aplicou a pressão de seleção por meio da diminuição do tempo de sedimentação, e a estabilização dessa concentração na fase final do experimento (fase 3). Pode-se notar também que, ao longo da fase 2 , houve perda de lodo no reator após cada ciclo de aumento de velocidade de sedimentação, o que indica que essa estratégia de seleção foi efetiva por proporcionar a eliminação das partículas com menor capacidade de sedimentação do reator. O comportamento da concentração de

Tabela 2 - Características físicas e químicas do afluente utilizado nos ensaios.

\begin{tabular}{|c|c|c|c|}
\hline Parâmetro & Unidade & $\begin{array}{c}\text { Média e } \\
\text { desvio padrão }\end{array}$ & $\begin{array}{c}\mathrm{N}^{\circ} \text { de } \\
\text { análises }\end{array}$ \\
\hline Oxigênio dissolvido & $\mathrm{mg} \cdot \mathrm{L}^{-1}$ & $1,50 \pm 0,54$ & 184 \\
\hline $\mathrm{pH}$ & - & $6,7 \pm 0,29$ & 184 \\
\hline $\mathrm{DQO}_{\text {total }}$ & $\mathrm{mg} \cdot \mathrm{L}^{-1}$ & $388,57 \pm 103,45$ & 37 \\
\hline $\mathrm{DQO}_{\text {solúvel }}$ & $\mathrm{mg} \cdot \mathrm{L}^{-1}$ & $233,70 \pm 81,48$ & 37 \\
\hline Sólidos totais & $\mathrm{mg} \cdot \mathrm{L}^{-1}$ & $852,70 \pm 369,02$ & 37 \\
\hline Sólidos totais fixos & $\mathrm{mg} \cdot \mathrm{L}^{-1}$ & $379,19 \pm 163,65$ & 37 \\
\hline Sólidos totais voláteis & $\mathrm{mg} \cdot \mathrm{L}^{-1}$ & $473,51 \pm 330,67$ & 37 \\
\hline Sólidos suspensos totais & $\mathrm{mg} \cdot \mathrm{L}^{-1}$ & $111,73 \pm 65,46$ & 37 \\
\hline Sólidos suspensos fixos & $\mathrm{mg} \cdot \mathrm{L}^{-1}$ & $27,09 \pm 24,55$ & 37 \\
\hline Sólidos suspensos voláteis & $m g \cdot L^{-1}$ & $84,63 \pm 55,95$ & 37 \\
\hline NTK & $\mathrm{mg} \cdot \mathrm{L}^{-1}$ & $80,36 \pm 11,78$ & 37 \\
\hline Nitrogênio amoniacal (N-NH$\left.{ }^{4+}\right)$ & $\mathrm{mg} \cdot \mathrm{L}^{-1}$ & $71,15 \pm 9,56$ & 37 \\
\hline
\end{tabular}

DQO: demanda química de oxigênio; NTK: nitrogênio total Kjeldahl.

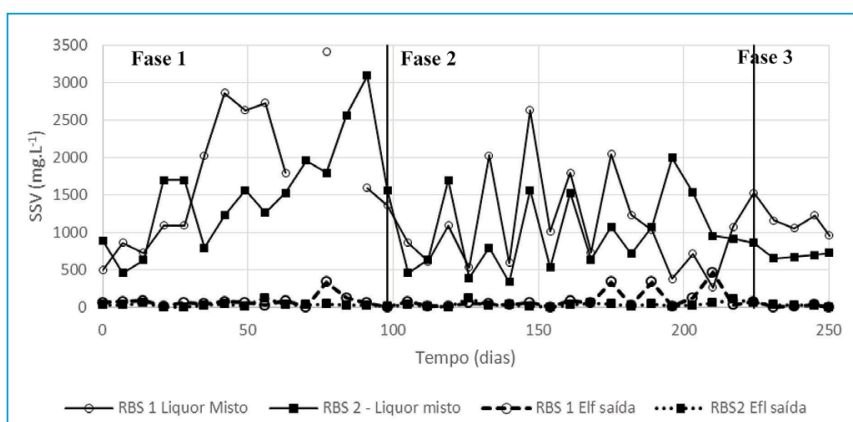

RBS: reator em batelada sequencial; SSV: sólidos suspensos voláteis; efl: efluente. Fonte: elaborada pelos autores

Figura 4 - Concentração de sólidos suspensos voláteis no liquor misto e no efluente de saída nos reatores em batelada sequencial 1 e 2 .
SSV no efluente da saída tanto do RBS 1 como do RBS 2 corrobora os resultados observados.

A avaliação do índice volumétrico de lodo (IVL) para os tempos 5 e 30 min (Figuras 5 e 6) é uma informação importante para verificar a característica hidrodinâmica das partículas constituintes do lodo (biomassa), refletindo diretamente a sua condição de retenção no reator. O valor do IVL, em mL.g ${ }^{-1}$, está associado diretamente à densidade dos flocos ou grânulos formados. A relação $\mathrm{IVL}_{30} / \mathrm{IVL}_{5}$, por sua vez, é um indicativo da velocidade de sedimentação das partículas, e, quanto mais próximo de $100 \%$, mais rapidamente se dá a sedimentação, tendo o lodo uma condição de retenção mais consistente e efetiva (LIU et al., 2010; LIU et al., 2011; WAGNER \& COSTA, 2013; CETIN et al., 2018).

Observa-se, da análise das Figuras 5 e 6, a diminuição do valor de IVL na fase de aclimatação do lodo, seguida por um período de instabilidade (fase 2) em ambos os reatores. Os valores elevados de IVL no início do experimento mostram que o lodo utilizado como inóculo tinha baixa capacidade de sedimentação em razão de ser oriundo de um sistema em que a separação entre a fase sólida e fase líquida era feita utilizando-se membranas. Com a imposição

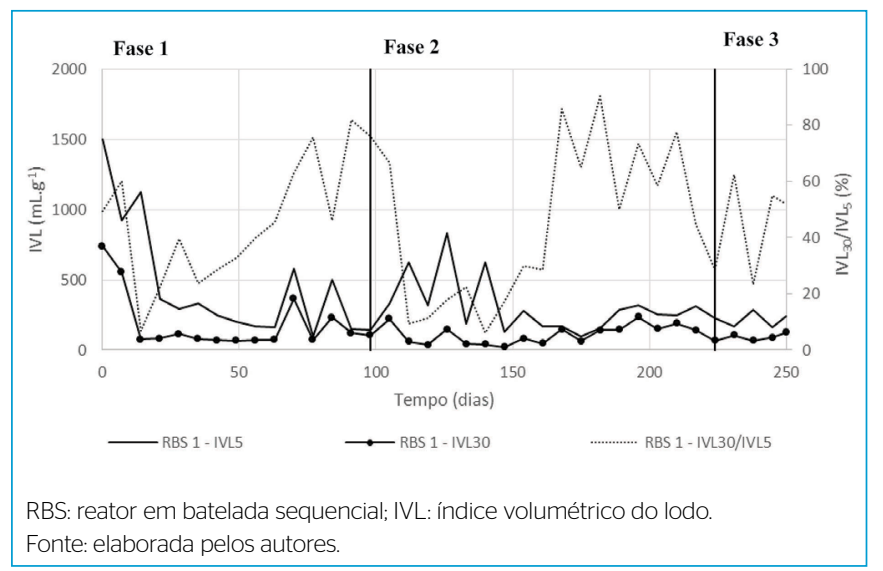

Figura 5 - Valores do índice volumétrico do lodo para os tempos de 5 e 30 minutos e da razão entre os esses mesmos índices para os tempos de 30 e 5 minutos, no reator de batelada sequencial 1 .

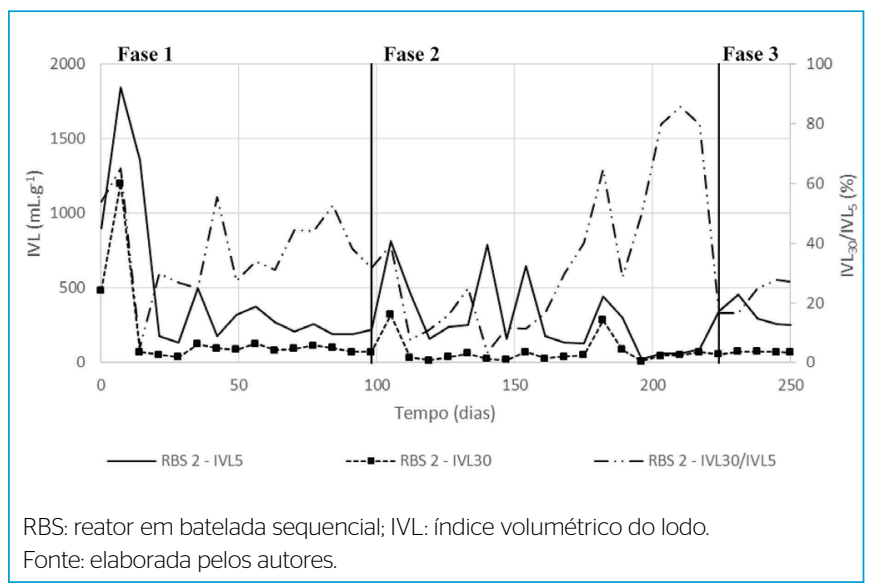

Figura 6 - Valores do índice volumétrico do lodo para os tempos de 5 e 30 minutos e da razão entre os esses mesmos índices para os tempos de 30 e 5 minutos, no reator de batelada sequencial 2 . 
do tempo de sedimentação de 10 min (dia 196), verifica-se a estabilização dos valores de IVL, com $\mathrm{IVL}_{30}$ inferiores a $100 \mathrm{~mL} \cdot \mathrm{g}^{-1}$.

Os dados contidos nas Figuras 5 e 6 foram avaliados por meio do teste $t$ de Student ao nível de significância de $\mathrm{p}=0,05$. Os resultados indicaram somente diferença significativa de comportamento entre os lodos dos reatores RBS $1 \mathrm{e}$ RBS 2 quando se compararam os valores para a relação $I_{V L} L_{30} / I L_{5}$ na fase 3 . Nesta fase, o RBS 1 apresentou o melhor desempenho para esse indicador, decorrente de valores um pouco superiores para a velocidade de sedimentação zonal do lodo, o que será discutido em detalhes a seguir.

Pelos valores de IVL observados em cada reator, pôde-se afirmar que a biomassa formada tinha boa capacidade de sedimentação. Contudo, os baixos valores relatados para a razão $\mathrm{IVL}_{30} / \mathrm{IVL}_{5}$, principalmente para o RBS 2, podem estar relacionados à produção de novos flocos/grânulos. Mesmo com o tempo de sedimentação considerado muito baixo, os novos flocos/grânulos produzidos permaneceram no reator em razão da baixa relação $H / D$, ou seja, mesmo com os grânulos apresentando velocidade considerada baixa para a sedimentação, o espaço percorrido até a sua estabilidade no fundo do reator era pequeno também.

Os valores verificados para esta relação, $\mathrm{IVL}_{30} / \mathrm{IVL}_{5}$, entretanto, são inferiores àqueles encontrados por outros autores, que se aproximam de $100 \%$ - como os apresentados por Liu e Tay (2012), em que a relação do IVL variou de 75 a 100\%, e por Liu et al. (2011), em que a relação variou entre 90 e 100\% nos últimos dias de operação. Os valores baixos da relação $\mathrm{IVL}_{30} / \mathrm{IVL}_{5}$ indicam que há prevalência de biomassa não granular no reator, apesar da presença de grânulos.

Na Figura 7 são apresentados os valores do diâmetro médio da biomassa no interior dos reatores, obtidos com a utilização de microscópio óptico e software de análise de imagens. É possível verificar pouca variação média no diâmetro médio da biomassa até o meio da fase 2, quando há uma diminuição desse diâmetro para ambos os reatores.

Nota-se também, ao se analisar a etapa final do experimento, fase 3 (Figura 7), que os grânulos formados eram pouco estáveis. O que se verifica, tanto para o RBS 1 quanto para o RBS 2, é o crescimento do diâmetro dos grânulos até os valores de 1,05 e 1,28 mm, respectivamente, e posterior diminuição. Esse comportamento pode ser o resultado da quebra desses grânulos maiores em razão da baixa estabilidade estrutural. Segundo Franca et al. (2017), ainda são muito pouco conhecidos e elucidados os mecanismos relativos à perda da estabilidade estrutural dos grânulos, sabendo-se por enquanto algumas condições operacionais que podem promover essa perda, entre elas presença de compostos tóxicos e períodos curtos de inanição.

A partir do dia 150, aproximadamente, inicia-se o processo de formação dos grânulos propriamente dito, com a substituição dos flocos de diâmetros maiores por grânulos de pequeno diâmetro, cujo tamanho tende a aumentar com o tempo. O aumento nas concentrações de EPS (Figura 8) indica que a aplicação da pressão de seleção foi suficiente para induzir a produção dessa substância, responsável pela formação, estabilidade e maior densidade dos grânulos (ADAV \& LEE, 2008; PAN et al., 2010; DING et al., 2015).

Em razão das dificuldades de extração do EPS em lodos granulares, bem como das baixas concentrações relativas de proteínas e carboidratos no extrato, os valores apresentados na Figura 8 devem ser analisados de forma qualitativa, isto é, eles mostram que, com a aplicação da pressão de seleção, há tendência de aumento da produção de EPS em ambos os reatores.

Nas Figuras 9 e 10 são apresentadas imagens das alterações ocorridas no aspecto da biomassa ao longo do tempo para os reatores RBS 1 e RBS 2, respectivamente. A escala gráfica indicada nas figuras (barra horizontal na parte inferior direita) tem comprimento de $1 \mathrm{~mm}$.

As imagens apresentadas nas Figuras 9 e 10 corroboram os resultados apresentados nas Figuras 7 e 8; com a aplicação da pressão de seleção e consequente produção de EPS, os flocos foram sendo substituídos por grânulos, de diâmetro pequeno, mas com tendência ao crescimento dessa dimensão. À medida que o diâmetro dos grânulos vai aumentando, eles perdem estabilidade e rompem-se, conforme fica claro nas Figuras 9F e 10D, nas quais é possível verificar a desintegração dos grânulos maiores, formando grânulos menores e com superfície irregular. Comportamento similar a esse foi relatado por Sengar et al. (2018), ao analisarem fotografias de microscopia extraídas de Basheer (2012).

Dessa forma, verifica-se que efetivamente ocorreu a formação de grânulos no interior dos reatores, apesar da baixa velocidade de sedimentação imposta, e que essa formação se deu ao longo da fase 2 do experimento.

Os resultados de ensaios de velocidade de sedimentação zonal do lodo são apresentados na Figura 11, junto dos valores de velocidade de sedimentação impostos. Observa-se, na figura em questão, que a velocidade de sedimentação do lodo nos dois reatores superou, ao longo de todo o experimento, as velocidades impostas. Na fase 1 , apesar de ser imposta uma velocidade de sedimentação

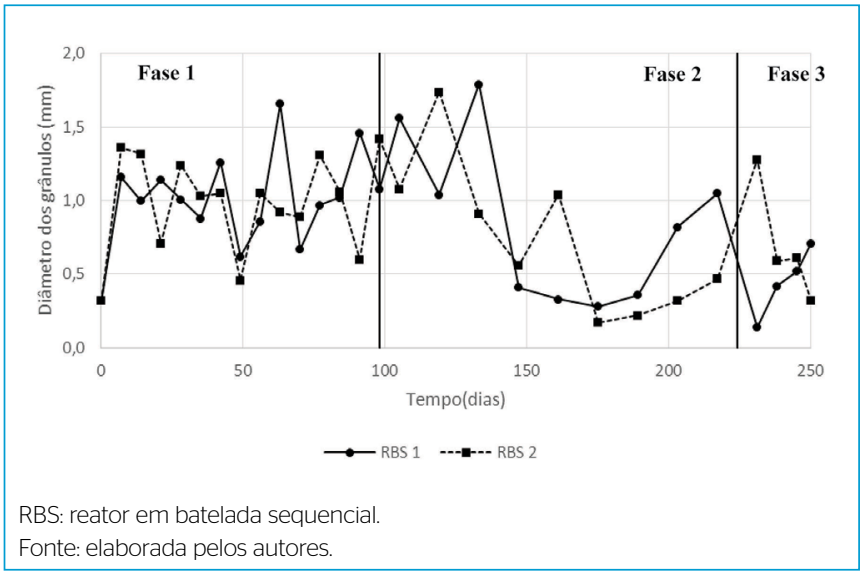

Figura 7 - Diâmetro médio dos grãos (flocos ou grânulos).

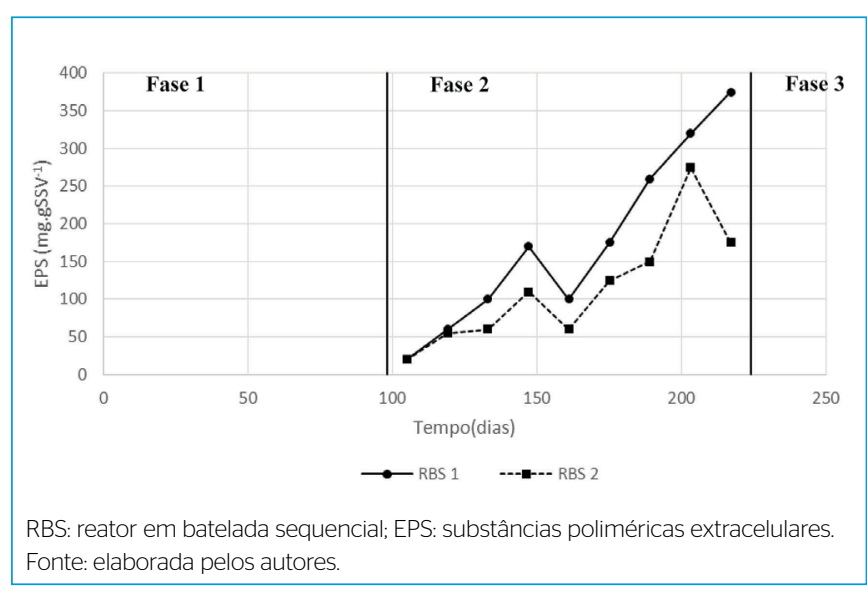

Figura 8 - Concentração de substância polimérica extracelular ao longo da fase 2. 
bastante baixa e constante, houve aumento na velocidade de sedimentação real do lodo, fato explicitado pelo acréscimo na velocidade de sedimentação zonal. Observa-se também que essa elevação na velocidade ocorre nos dois reatores, independentemente na RTV. Tal comportamento deve-se à baixa capacidade de sedimentação do inóculo, oriundo de um reator no qual se utilizavam membranas para a separação dos sólidos.

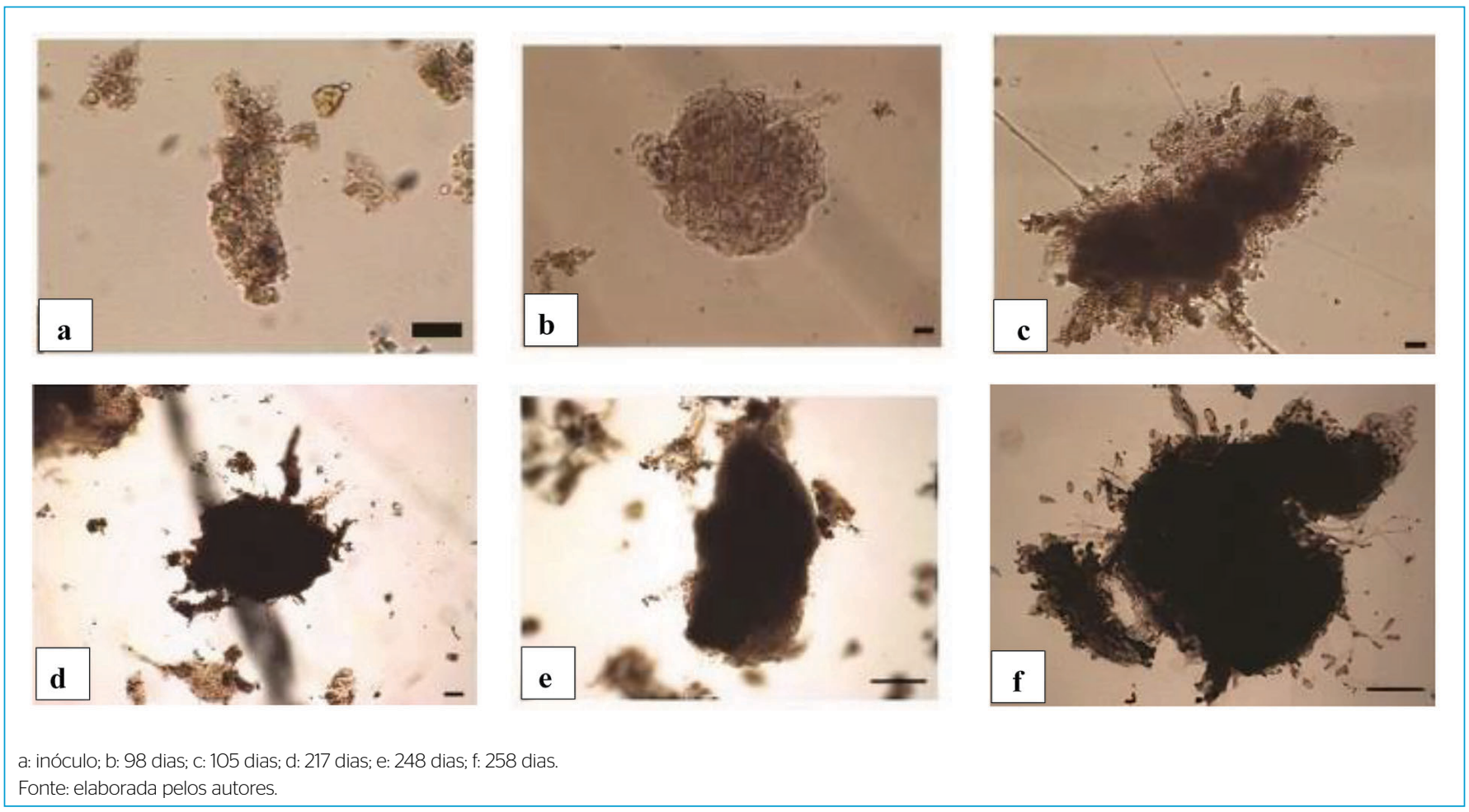

Figura 9 - Alterações na biomassa ao longo do tempo, para o reator em batelada sequencial 1.
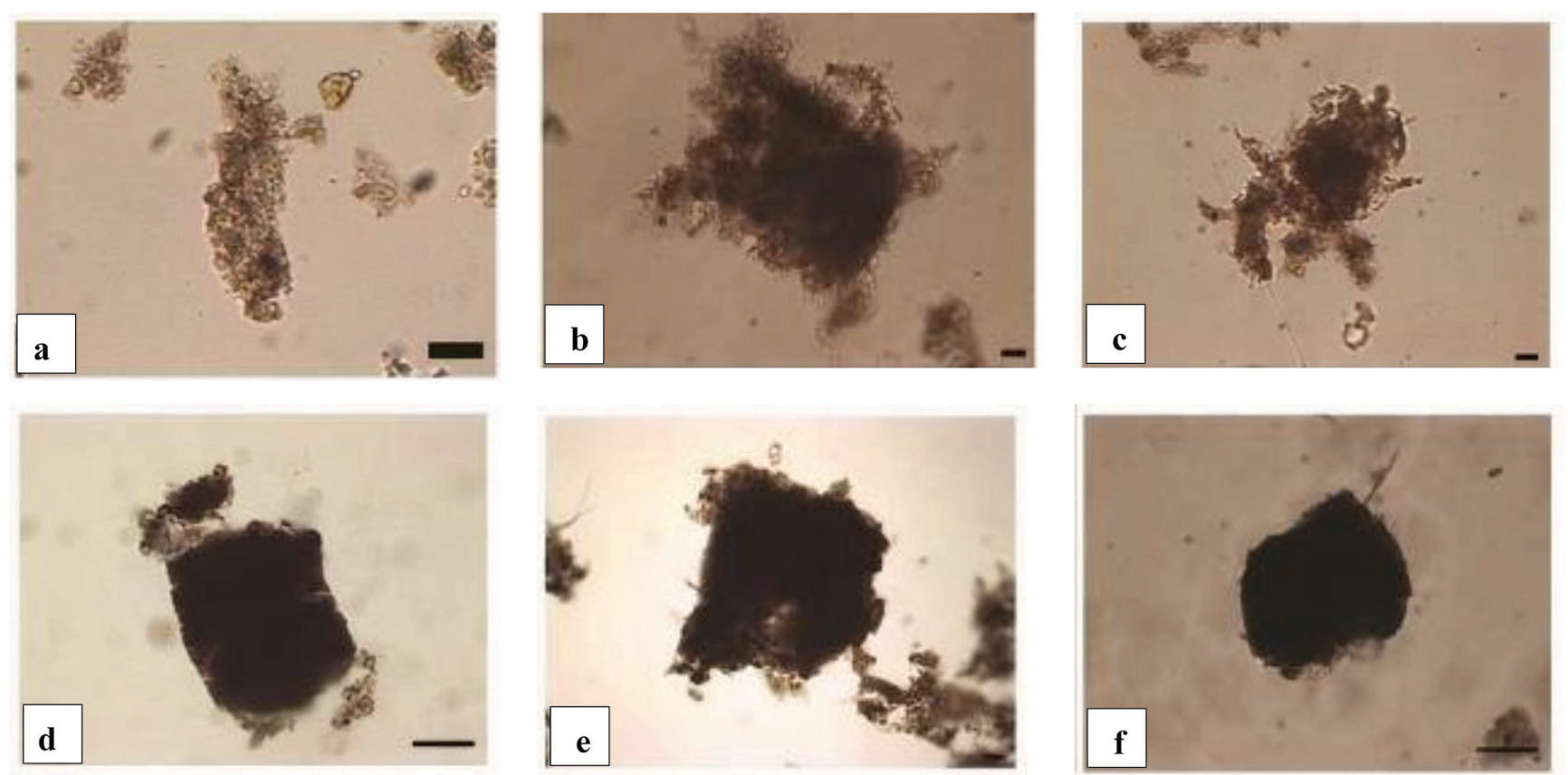

a: inóculo; b: 98 dias; c: 105 dias; d: 217 dias; e: 248 dias; f: 258 dias. Fonte: elaborada pelos autores.

Figura 10 - Alterações na biomassa ao longo do tempo, para o reator em batelada sequencial 2. 
O aumento na velocidade real das partículas parece se dar pelo acréscimo na sua densidade, posto que não há variação significativa no diâmetro. Ao se analisarem as Figuras 9A-9C e 10A-10C, pode-se verificar um incremento na densidade dos flocos, que se tornaram mais opacos. Esse aumento na densidade não foi causado pela aplicação de pressão de seleção advinda da velocidade de sedimentação. Entretanto, é importante salientar que o lodo estava submetido a forças de cisalhamento advindas da mistura existente na etapa anaeróbia e da aeração na etapa aeróbia. Essas forças parecem ter induzido a densificação das partículas, dado que as regiões mais externas dos flocos tendem a ser mais frágeis e menos densas.

Ao longo da fase 2, a velocidade de sedimentação real do lodo tendeu a se estabilizar em 2,5 m.h. ${ }^{-1}$ (Figura 11). Verifica-se, conforme já demonstrado anteriormente, que a formação dos grânulos se dá ao longo dessa fase, o que mostra, portanto, que foi possível produzir grânulos apesar de a velocidade de sedimentação real ser inferior aos valores reportados na literatura. Sendo assim, pode-se dizer que, para a situação em análise, a indução na formação do grânulo foi consequência, principalmente, da razão de crescimento da velocidade de sedimentação, decorrente da estratégia adotada na diminuição do tempo imposto à etapa de sedimentação.

Durante toda a fase 2, o tempo de sedimentação foi diminuído em 5 minutos a cada 14 dias de operação. No início esse valor era de 50 minutos e, ao fim, de 5 minutos. Como resultado, no início dessa fase, a fração de diminuição do tempo de sedimentação iniciou-se com o valor de $1 / 10$ e finalizou com o valor de 1/2, apresentando um comportamento exponencial de crescimento. Dessa forma, a pressão de seleção dependeu efetivamente da razão de crescimento imposta no aumento da velocidade de sedimentação, influenciando decisivamente no comportamento hidrodinâmico das partículas. Quando essa pressão de seleção foi aplicada de forma lenta e gradual, conforme se verificou no início da fase 2 , não houve resposta em relação à velocidade de sedimentação real do lodo, 0 que se deu somente no final dessa fase, quando a pressão de seleção foi imposta de forma mais efetiva em decorrência do aumento exponencial na velocidade de sedimentação imposta.

Ao se analisar o comportamento das concentrações de EPS na biomassa e a velocidade de seleção imposta ao longo da fase 2 , nota-se que há semelhança nas tendências de crescimento dessas duas variáveis para os dois reatores, indicando que o aumento da pressão de seleção imposta induziu à liberação de EPS

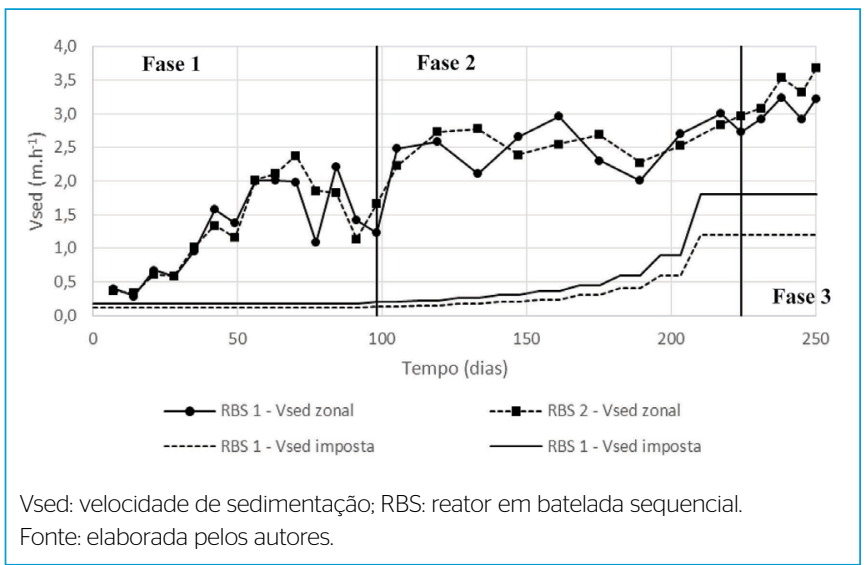

Figura 11 - Velocidade de sedimentação zonal e imposta, para os reatores em batelada sequencial 1 e 2 . pelos microrganismos. $\mathrm{O}$ aumento nas concentrações de EPS, entretanto, não influenciou na velocidade de sedimentação real dos grânulos. Acredita-se que, à medida que houve o acréscimo de EPS, tornando os grânulos mais densos, houve também o aumento no diâmetro desses grânulos, fato que contribui para $o$ aumento da resistência ao arraste. Assim, pode-se inferir que o aumento na densidade dos grânulos, decorrente da elevação das concentrações de EPS, foi equilibrado com a expansão do diâmetro, o que veio a provocar maior resistência de arraste aos grânulos. À medida que os grânulos foram sendo quebrados, no final da fase 2 , houve tendência ao aumento na velocidade de sedimentação real. É importante salientar que a ruptura dos grânulos, conforme se pode observar nas Figuras 9F e 10D, gera outros grânulos mais irregulares, que têm maior resistência ao arraste.

$\mathrm{Na}$ fase 3, por sua vez, os grânulos mantiveram as mesmas características verificadas ao fim da fase 2 com respeito à concentração de EPS e ao diâmetro médio. $\mathrm{O}$ aumento da esfericidade das partículas, perceptível na análise das Figuras 9E, 9F, 10E e 10F, parece ter contribuído para o acréscimo na velocidade de sedimentação.

A análise dos dados não permite apontar diferenças de comportamento quanto à formação dos grânulos entre os reatores RBS 1 e RBS 2. Isso indica que, para este experimento, a RTV não foi uma variável importante na indução da formação da biomassa granular.

\section{EFICIÊNCIA DE REMOÇÃO DE MATÉRIA ORGÂNICA E NITROGẾNIO}

Na Tabela 3 são apresentados valores para as concentrações e para as eficiências de remoção de matéria orgânica $\left(\mathrm{DQO}_{\text {solúvel }}\right)$ e nitrogênio total Kjeldahl (NTK) ao longo do tempo, respectivamente, para os reatores RBS 1 e RBS 2.

Verifica-se na fase 1, enquanto a biomassa estava na forma de flocos, que as eficiências de remoção de carbono e nitrogênio foram relativamente elevadas após o dia 20, aproximadamente, alcançando valores médios de remoção de $75 \%$ para a matéria orgânica solúvel e de $80 \%$ para o nitrogênio, na forma amoniacal e NTK. Verifica-se também comportamento similar entre os reatores RBS1 e RBS2.

Observando as Figuras 12 e 13, vê-se que o comportamento do RBS 2 para a $\mathrm{DQO}_{\text {soluvel }}$ foi diferente quando comparado ao RBS 1. Como a razão de troca volumétrica foi menor no segundo reator, a carga orgânica volumétrica (COV) foi baixa. Assim, as concentrações do afluente eram baixas, logo as bactérias do RBS 2 assimilaram o substrato, gerando efluentes com baixas concentrações.

Observando-se os valores da concentração de $\mathrm{DQO}_{\text {soluvel }}$ nos efluentes dos RBS1 e RBS2 e as respectivas eficiências de remoção (Tabela 3 e Figuras 12 e 13), não foram verificadas diferenças significativas ao nível de significância de $\mathrm{p}=0,05$ para a análise de dados pareados (utilizando o teste $t$ de Student) para as três fases de operação. Contudo, o comportamento do RBS1 indicou maiores valores de eficiência de remoção nas fases 2 e 3, em que o maior valor de RTV possibilitou apenas um pequeno aumento na eficiência de remoção, porém com melhores resultados na formação de grânulos no lodo.

A segunda fase foi marcada por apresentar uma variação da eficiência decorrente da diminuição do tempo de sedimentação ao longo do experimento. Ao reduzir-se o tempo de sedimentação durante as semanas, a concentração de sólidos orgânicos variou, ocasionando a lavagem do lodo de baixa sedimentabilidade. 
Tabela 3 - Resultados da concentração e da eficiência de remoção para demanda química de oxigênio da fração solúvel e nitrogênio total Kjeldahl das amostras do sistema.

\begin{tabular}{|c|c|c|c|c|c|c|c|c|c|c|c|}
\hline \multirow{2}{*}{ Amostra } & \multirow{2}{*}{ Parâmetro } & \multirow{2}{*}{ Unidade } & \multicolumn{3}{|c|}{ Fase 1} & \multicolumn{3}{|c|}{ Fase 2} & \multicolumn{3}{|c|}{ Fase 3} \\
\hline & & & $\mathrm{x}$ & s & $\mathrm{n}$ & $\mathrm{x}$ & s & $\mathrm{n}$ & $\mathrm{x}$ & s & $\mathrm{n}$ \\
\hline Afluente & $\mathrm{DQO}_{\text {solível }}$ & $\mathrm{mg} \cdot \mathrm{L}^{-1}$ & 254,64 & 73,74 & 14 & 230,89 & 90,04 & 18 & 185,20 & 55,74 & 5 \\
\hline \multirow{2}{*}{ Efl saída RBS 1} & $\mathrm{DQO}_{\text {solivel }}$ & mg. L $^{-1}$ & 85,00 & 73,10 & 14 & 59,11 & 21,25 & 18 & 62,00 & 17,85 & 5 \\
\hline & NTK & $m g . L^{-1}$ & 31,60 & 21,63 & 13 & 38,84 & 19,71 & 18 & 24,53 & 8,35 & 5 \\
\hline Efl saída RBS 2 & NTK & $\mathrm{mg} \cdot \mathrm{L}^{-1}$ & 22,91 & 21,49 & 13 & 25,58 & 15,59 & 18 & 38,18 & 16,65 & 5 \\
\hline \multirow{2}{*}{ Remoção RBS 1} & $\mathrm{DQO}_{\text {solível }}$ & $\%$ & 67,52 & 19,77 & 14 & 71,77 & 12,91 & 18 & 61,04 & 24,33 & 5 \\
\hline & NTK & $\%$ & 60,47 & 26,14 & 13 & 51,63 & 20,50 & 18 & 73,58 & 9,44 & 5 \\
\hline \multirow{2}{*}{ Remoção RBS 2} & $\mathrm{DQO}_{\text {solúvel }}$ & $\%$ & 71,33 & 12,50 & 14 & 65,12 & 17,00 & 18 & 56,98 & 18,47 & 5 \\
\hline & NTK & $\%$ & 72,16 & 25,77 & 13 & 68,01 & 17,14 & 18 & 59,18 & 17,70 & 5 \\
\hline
\end{tabular}

$\mathrm{DQO}_{\text {solível: }}$ demanda química de oxigênio da fração solúvel; NTK: nitrogênio total Kjeldahl; * X: valor médio; s: desvio padrão; n: número de amostras; RBS: reator em batelada sequencial; efl: efluente.

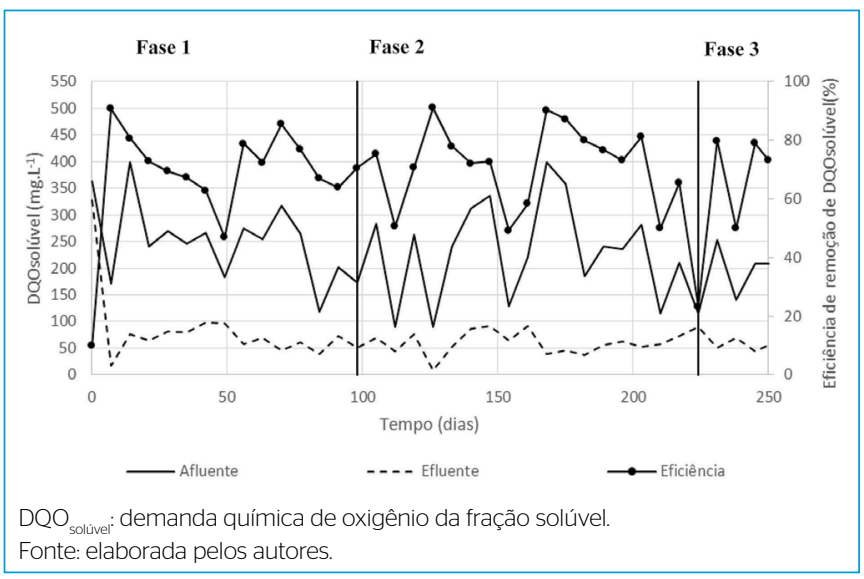

Figura 12 - Concentração e eficiência de remoção de matéria orgânica do reator em batelada sequencia 1 .

Comparando-se os valores da concentração de NTK nos efluentes dos RBS1 e RBS2 e as respectivas eficiências de remoção (Tabela 3), não foram verificadas diferenças significativas ao nível de significância de $\mathrm{p}=0,05$ para a análise de dados pareados (utilizando o teste $t$ de Student) para as fases 1 e 3 de operação. Para a fase 2 ocorreu diferença significativa entre os reatores, e o RBS2 apresentou melhor desempenho.

$\mathrm{Na}$ fase 2, verifica-se maior instabilidade nos resultados em virtude da aplicação da pressão de seleção, principalmente na remoção do nitrogênio. $\mathrm{Na}$ fase 3, verifica-se que, apesar da formação consistente de biomassa granular, os resultados de eficiência de remoção ainda não estavam estáveis, provavelmente em decorrência da baixa estabilidade dos grânulos formados nos reatores.

De forma geral, pode-se afirmar que a RTV não teve influência na eficiência de remoção de matéria orgânica e nitrogênio. Conforme relatado por Wagner et al. (2015), Liu et al. (2010) e Liu et al. (2011), a maior degradação da matéria orgânica e do nitrogênio dá-se no início do ciclo, estabilizando-se após aproximadamente 1 hora de reação aeróbia. Dessa forma, apesar de o reator com maior RTV ter sido submetido a maior carga de matéria orgânica

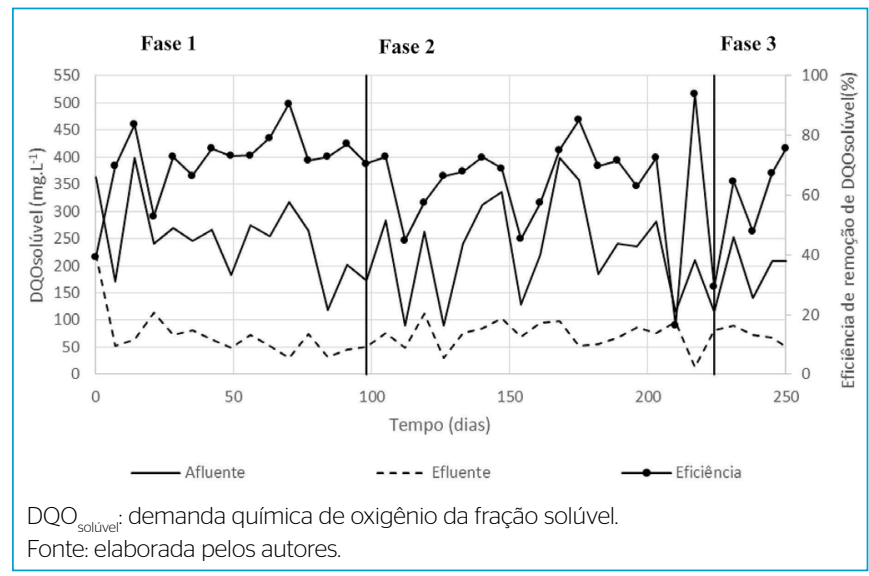

Figura 13 - Concentração e eficiência de remoção de matéria orgânica do reator em batelada sequencia 2 .

e nitrogênio, em razão do elevado tempo de ciclo utilizado no experimento, ocorria um período prolongado de famine para ambos os reatores. Diferenças significativas de eficiência poderiam ser observadas para ciclos mais curtos, em que o tempo de reação poderia ser decisivo nesse comportamento.

\section{CONCLUSÕES}

Este trabalho mostrou que é possível a formação de biomassa granular aeróbia em reatores com velocidades de sedimentação baixas $\left(1,8\right.$ e $\left.1,2 \mathrm{~m} \cdot \mathrm{h}^{-1}\right) \mathrm{e}$ relação H/D unitária. Verificou-se que as velocidades de sedimentação real do lodo foram superiores àquelas impostas nos reatores ao longo de todo o experimento, mas que, independentemente disso, a estratégia de pressão de seleção aplicada foi suficiente para promover a retirada das partículas com menor capacidade de sedimentação do reator e para o estímulo da produção de EPS pelas bactérias.

$\mathrm{O}$ aumento da velocidade de sedimentação na fase inicial do experimento deu-se em razão do aumento na densidade das partículas. A partir da aplicação da pressão de seleção (fase 2), houve produção de EPS e a formação dos 
Não houve influência significativa da RTV nas eficiências de remoção de matéria orgânica e nitrogênio nem na formação do LGA.

\section{CONTRIBUIÇÃO DOS AUTORES}

Pires, D. P.: conceituação, curadoria de dados, análise formal, metodologia, escrita - primeira redação, revisão e edição. Benatti, J. C. B.: conceituação, análise formal, escrita - primeira redação, revisão e edição. Nour, E. A. A.: conceituação, supervisão, escrita - revisão e edição.

\section{REFERÊNCIAS}

ADAV, S. S.; LEE, D. J. Extraction of extracellular polymeric substances from aerobic granule with compact interior structure. Journal of Hazard Mater, v. 154, n. 1-3, p. 1120-1126, 2008. https://doi.org/10.1016/j.jhazmat.2007.11.058

AWANG, N. A., SHAABAN, M. G. Effect of reactor height/diameter ratio and organic loading rate on formation of aerobic granular sludge in sewage treatment. International Biodeterioration \& Biodegradation, v. 112, p. 1-11, 2016. https://doi.org/10.1016/j.ibiod.2016.04.028

APHA; AMERICAN WATER WORKS ASSOCIATION (AWWA); WEF. Standard methods for the examination of water and wastewater. $2^{19}$ ed. Washington: LMC - Pharmabooks, 2005.

AKABOCI, T. R. V. Tratamento de esgoto sanitário em reator em bateladas sequenciais: desempenho do processo e modelagem matemática. 169 p. Dissertação (Mestrado em Engenharia Ambiental) - Universidade Federal de Santa Catarina, Florianópolis, 2013.

BASHEER, F. Performance of sequencing batch reactor for the biodegradation of phenolic compounds. 176 p. Tese (Doutorado em Engenharia Civil) - Department of Civil Engineering Z.H., College of Engineering and Technology, Aligarh Muslim University, 2012

BUENO, R. F; SUBTIL, E. L.; GASPAR, J.; INÁCIO, A. R.; MOURÃO, R. F. Caracterização de flocos de lodo ativado por meio de análise de imagem: padronização e validação do método. In: 270 Congresso Brasileiro de Engenharia Sanitária e Ambiental, 2013, Goiânia. 270 CBESA, 2013.

CETIN, E.; KARAKAS, E.; DULEKGURGEN, E.; OVEZ, S.; KOLUKIRIK, M.; YILMAZ, G. Effects of high-concentration influent suspended solids on aerobic granulation in pilot-scale sequencing batch reactors treating real domestic wastewater. Water Research, v. 131, p. 74-89, 2018. https://doi. org/10.1016/j.watres.2017.12.014

CORSINO, S. F.; CAMPO, R.; DI BELLA, G.; TORREGROSSA, M.; VIVIANI, G. Study of aerobic granular sludge stability in a continuous-flow membrane bioreactor. Bioresource Technology, v. 200, p. 1055-1059, 2016. https://doi. org/10.1016/j.biortech.2015.10.065

DE KREUK, M. K.; KISHIDA, N.; VAN LOOSDRECHT, M. C.M. Aerobic granular sludge-state of the art. Water Science and Technology, v. 55, n. 8-9, p. 75-81, 2007. https://doi.org/10.2166/wst.2007.244

DING, Z., BOURVEN, I., GUIBAUD, G., VAN HULLEBUSCH, E. D., PANICO, A., PIROZZI, F., ESPOSITO, G. Role of extracellular polymeric substances
(EPS) production in bioaggregation: application to wastewater treatment. Applied Microbiology and Biotechnology, v. 99, p. 9883-9905, 2015. https:// doi.org/10.1007/s00253-015-6964-8

FRANCA, R. D. G.; PINHEIRO, H.M.; VAN LOOSDRECHT, M. C. M.; LOURENÇO, N. D. Stability of aerobic granules during long-term bioreactor operation. Biotechnology Advances, v. 36, n. 1, p. 228-246, 2017. https://doi.org/10.1016/j. biotechadv.2017.11.005

HE, Q.; ZHOU, J.; WANG, H.; ZHANG, J.; WEI, L. Microbial population dynamics during sludge granulation in an $\mathrm{A} / \mathrm{O} / \mathrm{A}$ sequencing batch reactor. Bioresource Technology, v. 214, p. 1-8, 2016. https://doi.org/10.1016/j. biortech.2016.04.088

HE, Q.; ZHANG, W.; ZHANG, S.; HOUGYU, W. Enhanced nitrogen removal in an aerobic granular sequencing batch reactor performing simultaneous nitrification, endogenous denitrification and phosphorus removal with low superficial gas velocity. Chemical Engineering Journal, v. 326, p. 1223-1231, 2017. http://doi.org/10.1016/j.cej.2017.06.071

HE, Q.; SONG, Q.; ZHANG, S.; ZHANG, W.; WANG, H. Simultaneous nitrification, denitrification and phosphorus removal in an aerobic granular sequencing batch reactor with mixed carbon sources: reactor performance, extracellular polymeric substances and microbial successions. Chemical Engineering Journal, v. 331, p. 841-849, 2018. https://doi.org/10.1016/j. cej.2017.09.060

JUNGLES, M. K. Desenvolvimento de grânulos aeróbios no tratamento de esgoto sanitário em reatores operados em bateladas sequenciais (RBS). Tese (Doutorado em Engenharia Ambiental) - Universidade Federal de Santa Catarina, Florianópolis, SC, 2011.

LI, D.; ZHANG, S.; LI, S.; ZENG, H.; ZHANG, J. The nitrogen removal of autotrophic and heterotrophic bacteria in aerobic granular reactors with different feast/famine ratio. Bioresource Technology, v. 272, p. 370-378, 2019. https://doi.org/10.1016/j.biortech.2018.10.046

LIU, H.; FANG, H. H. P. Extraction of extracellular polymeric substances (EPS) of sludges. Journal of Biotechnology, v. 95, n. 3, p. 249-256, 2002. https://doi. org/10.1016/S0168-1656(02)00025-1

LIU, J.; LI, J.; WANG, X.; ZHANG, Q.; LITTLETON, H. Rapid aerobic granulation in an SBR treating piggery wastewater by seeding sludge from a municipal WWTP. Journal of Environmental Sciences, v. 51, p. 332-341, 2017. https://doi. org/10.1016/j.jes.2016.06.012 
LIU, Y.; WANG, Z.W.; QIN, L.; LIU, Y.-Q.; TAY, J.-H. Selection pressure-driven aerobic granulation in a sequencing batch reactor. Applied Microbiology and Biotechnology, v. 67, p. 26-32, 2005. https://doi.org/10.1007/s00253004-1820-2

LIU, Y.; TAY, J.-H. State of the art of biogranulation technology for wastewater treatment. Biotechnology Advances, v. 22, n. 7. p. 533-563, 2004. https://doi. org/10.1016/j.biotechadv.2004.05.001

LIU, Y.-Q.; MOY, B.; KONG, Y.H.; TAY, J.H. Formation, physical characteristics and microbial community structure of aerobic granules in a pilot-scale sequencing batch reactor for real wastewater treatment. Enzyme and Microbial Technology, v. 46, n. 5, p. 520-525, 2010. https://doi.org/10.1016/j. enzmictec.2010.02.001

LIU, Y.-Q.; KONG, Y.; TAY, J.-H.; ZHU, J. Enhancement of start-up of pilotscale granular SBR fed with real wastewater. Separation and Purification Technology, v. 82, p. 190-196, 2011. http://doi.org/10.1016/j.seppur.2011.09.014

LIU, Y.Q.; TAY, J.H. The competition between flocculent sludge and aerobic granules during the long-term operation period of granular sludge sequencing batch reactor. Environmental Technology, v. 33, n. 23, p. 26192626, 2012. https://doi.org/10.1080/09593330.2012.673011

LOWRY, O. H.; ROSEBROUGH, N. J.; FARR, A. L.; RANDALL, R. J. Protein measurement with the Folin phenol reagent. The Journal of Biological Chemistry, v. 193, n. 1, p. 265-275, 1951.

METCALF; EDDY INC. Wastewater Engineering: Treatment and Resource Recovery. $5^{a}$ ed. New York: McGraw-Hill International Editions, 2013.

NANCHARAIAH, Y. V.; KIRAN KUMAR REDDY, G. Aerobic granular sludge technology: Mechanisms of granulation and biotechnological applications. Bioresource Technology, v. 247, p. 1128-1143, 2018. https://doi.org/10.1016/j. biortech.2017.09.131

NIELSEN, S. S. Phenol-Sulfiric Acid Method for Total Carbohydrates. In: Food Analysis Laboratory Manual. 2a ed. West Lafayette: Springer Science+Business Media, 2010. p. 48-53.

PAN, X.; LIU, J.; ZHANG, D.; CHEN, X.; LI, L.; SONG, W.; YANG, J. A comparison of five extraction methods for extracellular polymeric substances (EPS) from biofilm by using threedimensional excitation-emission matrix (3DEEM) fluorescence spectroscopy. Water AS, v. 36, n. 1, p. 111-116, 2010. https://doi.org/10.4314/wsa.v36i1.50914

PUJOL, S. B. Biogrânulos aeróbicos em reator sequencial em batelada para o tratamento de efluente de biodigestor proveniente da suinocultura. 94 p. Dissertação (Mestrado em Ciências do Solo) - Universidade Federal de Santa Maria, Santa Maria, RS, 2008.
QIN, L.; LIU, Y.; TAY, J. H. Effect of settling time on aerobic granulation in sequencing batch reactor. Biochemical Engineering Journal, v. 21, n. 1, p. 4752, 2004. http://doi.org/10.1016/j.bej.2004.03.005

ROCKTÄSCHEL, T.; SOERENSE, K. H.; KLARMANN, C.; OCHOA, J. BOISSON, $\mathrm{P}$.; HORN, $\mathrm{H}$. Influence of the granulation grade on the concentration of suspended solids in the effluent of a pilot scale sequencing batch reactor operated with aerobic granular sludge. Separation and Purification Technology, v. 142, p. 234-241, 2015. http://doi.org/10.1016/j. seppur.2015.01.013

SCHWARZENBECK, N.; ERLEY, R.; WILDERER, P. A. Aerobic granular sludge in an SBR-system treating wastewater rich in particulate matter. Water Science and Technology, v. 49, n. 11-12, p. 41-46, 2004. https://doi.org/10.2166/ wst.2004.0799

SENGAR, A.; BASHEER, F.; AZIZ, A.; FAROOQI, I. H. Aerobic granulation technology: Laboratory studies to full scale practices. Journal of Cleaner Production, v. 197, p. 616-632, 2018. https://doi.org/10.1016/j. jclepro.2018.06.167

SHOW, K. Y.; LEE, D. J; TAY, J. H. Aerobic Granulation: Advances and Challenges. Appl Biochem Biotechnol, v. 167, n. 6. p. 1622-1640, 2012. https:// doi.org/10.1007/s12010-012-9609-8

STURM, M. B. S.; IRVINE, L. Dissolved oxygen as key parameter to aerobic granule formation. Water Science \& Technology, v. 58, n. 4, p. 781-787, 2008. https://doi.org/10.2166/wst.2008.393

SU, K. Z; NI, B. J; YU, H. Q. Modeling and Optimization of granulation process of activated sludge in sequencing batch reactors. Biotechnol and Bioengineering, v. 110, n. 5. p. 1312-1322, 2013. https://doi.org/10.1002/bit.24812

VON SPERLING, M. Lodos Ativados. Vol 1, 3a ed. Belo Horizonte: DESAUFMG, 2012

WAGNER, J.; DA COSTA, R. H. R. Aerobic Granulation in a Sequencing Batch Reactor Using Real Domestic Wastewater. Journal of Environmental Engineering, v. 139, n. 11, p. 1391-1396, 2013. https://doi.org/10.1061/(asce) ee.1943-7870.0000760

WAGNER, J.; GUIMARÃES, L. B.; AKABOCI, T. R. V.; COSTA, R. H. R. Aerobic granular sludge technology and nitrogen removal for domestic wastewater treatment. Water Science \& Technology, v. 71, n. 7. p. 1040-1046, 2015. https:// doi.org/10.2166/wst.2015.064

WANG, F.; YANG, F. L.; ZHANG, X. W.; LIU, Y. H.; ZHANG, H. M.; ZHOU, J. Effects of cycle time on properties of aerobic granules in sequencing batch airlift reactor. World Journal of Microbiology \& Biotechnology, v. 21, p. 1379-1384, 2005. https://doi.org/10.1007/s11274-005-5451-2 